\title{
A Socio-technical Approach for Transient SME Alliances
}

\author{
Yacine Rezgui \\ School of Engineering, Cardiff University, Queen's Buildings, \\ The Parade, Cardiff CF24 3AA, Wales, UK \\ RezguiY@cardiff.ac.uk
}

\begin{abstract}
The paper discusses technical requirements to promote the adoption of alliance modes of operation by SMEs in the construction sector. These requirements have provided a basis for specifying a set of functionality to support the collaboration and cooperation needs of SMEs. While service-oriented architectures and semantic web services provide the middleware technology to implement the identified functionality, a number of key technical limitations have been identified, including lack of support for the dynamic and non-functional characteristics of SME alliances distributed business processes, lack of execution monitoring functionality to manage running business processes, and lack of support for semantic reasoning to enable SME business process service composition. The paper examines these issues and provides key directions for supporting SME alliances effectively.
\end{abstract}

Keywords: SME, Alliance, Construction, Service Oriented Architecture.

\section{Introduction}

The past two decades have seen a change in all industries and businesses from organizations that are rigid to a more sub-contracting and partnering way of working, with a strong tendency to outsource production and ideas development [1,2]. In this context, Construction SMEs face a number of challenging issues [3, 2]:

- They tend to follow conventional business models that provide traditional products and services against cost competitiveness. There is little room for value added business services that differentiate SMEs.

- They are small independent organizations but many are highly dependent on larger industry players for their work. This can disadvantage SMEs since they can be regarded as only "piece-workers" to do tasks with little opportunity to add value.

- They tend only to be present in a project during their part of the activity and this discontinuity of involvement is a particular challenge for the industry in relation to the adoption of the right business models and modes of project operation.

The paper argues that the adoption of alliance modes of operations would (a) help create opportunities that are not found in traditional organizations; and (b) promote innovation with the potential for SMEs to respond to complex business environments. 
While the technological infrastructure necessary to support virtual business operations is now readily available $[4,5,6,7]$, the paper argues that SMEs require collaborative solutions sensitive to socio-cultural and organizational issues that underpin long-term ventures. This forms the research gap that the present paper is addressing.

First, the paper reviews current developments in virtual teams, service-oriented architectures, and collaborative solutions in the construction sector. The specification of the technological solution to support SME alliances is then given. This is followed by a discussion of existing limitations that hinder full adoption of the proposed serviceoriented solution. Finally, recommendations for future work are given.

\section{Related Work}

A number of concepts related to virtual teams have been reported in the literature, including: Virtual Enterprise (VE), virtual corporation, virtual organization, and alliance. While virtual organization and virtual corporation tend to refer to the same concept [7], Goranson [8] draws important differences between the virtual enterprise and virtual organization (or corporation), noting that the term 'corporation' suggests that there is an inherent vision of corporate identity. Enterprise conveys the meaning that the shared focus is the project at hand. Corporation implies a conventional organization whose control is centralized. Hence, the virtual enterprise is unified by its mission and distributed goals, not its control system [7]. An alliance sits in between a virtual enterprise and a virtual organization, as it has a corporate dimension but resembles the virtual enterprise in the way business is conducted.

Alliance arrangements rely on team working. A team is defined by (a) its unity of purpose, (b) its identity as a social structure, and (c) its members' shared responsibility for outcomes [4]. The distinctive characteristics of virtual teams include the fact that they are geographically, organizationally, and /or time, dispersed collections of individuals who rely primarily on ICTs to accomplish one or more organizational tasks [5]. They tend to be assembled to respond to specific business needs or customer demands [6].

Moreover, an alliance is defined as a grouping of partners built around a number of principles underpinned by the following key features [9]:

- A business model that provides sound controls whilst facilitating flexibility and innovation at the appropriate times. Legal, contractual and cultural diversity issues taken fully into account.

- A set of clearly defined but customizable roles.

- A total lifecycle process driven philosophy where the intervention of each SME in the alliance complements and provides added value across the supply chain while delivering customized services and / or products to customers' and clients'.

- A performance driven and environmental friendly approach to addressing clients' and customers' requirements.

- Technology and ICT mechanisms, underpinned by a sound legal and contractual framework, to support the operation and collaboration needs of the alliance (though appropriate to alliance, partner and project circumstances and needs). 
Rezgui and Miles [9] argue that an alliance of independent peer organizations requires a "management" hub through an alliance broker. This would ensure (a) co-ordination of alliance strategic direction, (b) marketing the alliance, (c) handling contractual and advising on legal issues, (d) negotiating common resources (e.g. insurance and equipment), and (e) managing business opportunities. The latter is initially a bridgehead to customers that enquire but becomes the communication channel between the alliance and the appropriate partners for the particular opportunity, including their selection from the cluster of partners in the alliance. It is perceived as a brokering role. The broker will ensure that an integrated approach to addressing construction industry key challenges, including energy savings, is adopted. Such an approach coordinates technical and policy solutions, integrating engineering approaches with architecture, considering design decisions within the realities of building operation.

From a technological (software) perspective, the involvement in long-term partnerships with other SMEs in the context of alliances requires a shift from a software focused integration approach to a total lifecycle process integration philosophy where data / information / knowledge flows seamlessly. In this context, SMEs require focused, pragmatic, and easy to use solutions. Existing solutions tend to be technology (ICT) driven and are less sensitive to the socio-cultural and organizational issues that underpin long-term collaborative ventures.

SME characteristics and needs have to be factored into any collaborative platform for the concept of alliance to work. This platform must be able to transcend traditional methods based mainly on stable / static business models to facilitate dynamic alliances. Hence, there is a need to develop collaborative solutions addressing the needs of long-lasting alliances as opposed to existing solutions that suit the needs of large organizations collaborating with sub-contractors. Obviously, the two approaches are not mutually exclusive, but the former needs to be more sensitive to SME needs. A detailed discussion of SME socio-technical needs can be found in [9].

Web services have emerged as the most promising technology to provide the middleware platform to support effectively complex business operations [10, 11, 12, 13]. For many years, the goal towards which much research effort has been directed, concerns Service-oriented process implementations (or their ICT equivalent - business oriented service orchestrations) $[14,15]$ being able to transparently adapt to environment changes, including customer requirements, with minimal user intervention. As Leymann [16] notes, in current approaches, business process planning and implementation takes place during design-time when the architecture and the design of the software system are planned. The components to be used are chosen, linked together, and finally compiled and deployed. This approach is effectively the current state of the art in web services business process modeling practice and is successfully used and as long as the web service environment (business partners and service components) does not, or only rarely, changes.

However, if other businesses provide newer services, or other services replace older services, it is likely that inconsistencies may occur. In this case, an inevitable consequence is that the software architecture must be changed in order to accommodate new services (and their bindings to other services) or in the worst case, the process definition and design of the system needs to be changed. In fact, ACID (atomicity, consistency, integrity, and durability) properties of web service transactions are difficult to ensure [17]. In this context, static composition of services may be seen as 
restrictive, as components should ideally be able to automatically adapt to this kind of unpredictable change.

\section{Functionality Specification for Transient SME Alliances}

Based on the above, a number of technical and process requirements have been identified to support SME alliances [9]. Three categories of functionality emerge (Figure 1), namely:

Functionality to support alliance operations management:

$>$ Alliance formation and membership management: SME member identification, registration, profile definition, and membership validation / acceptance and management.

> Alliance nurturing and long-term management: functionality to support social networking within the alliance, promoting trust and shared responsibility management, supporting negotiation and conflict resolution, and functionality to support financial incentives and reward management.

$>$ Alliance creation and operations management: functionality for roles and responsibilities such as participation in decision-making, SME safeguards regarding information \& knowledge, resource scheduling in an alliance, and arrangements for cost-effective software use in SME alliances.

Functionality to support alliance business management:

$>$ Customer relationship management: integrated, socially-oriented approach to managing customers.

$>$ Business Opportunity Management: functionality to exploit business niches in the surrounding local, regional, and international business environment, and identify / nurture business opportunities.

$>$ Alliance branding and marketing: functionality to sustain a vibrant and positive (with a focus on sustainability and social corporate responsibility issues) outfacing image of the alliance.

$>$ Broker / Customer management: functionality supporting the SME Broker in channels of communication inside the alliance Virtual Factory and outside to customers, regulators, suppliers etc.

Functionality to support Alliance Capability, Learning, and Innovation Management

$>$ Functionality to support and nurture knowledge sharing and creation, including best practice.

$>$ Functionality to support awareness raising through push and / or pull mechanisms (dissemination / advertisement / broadcasting of SME information days, brochures, newsletters, etc.).

$>$ Innovation management environments including legal, IPR, contractual and cultural diversity issues.

$>$ Functionality to identify SME Learning and Training needs.

$>$ Functionality for SME learning and training needs learning / training modules definition. 


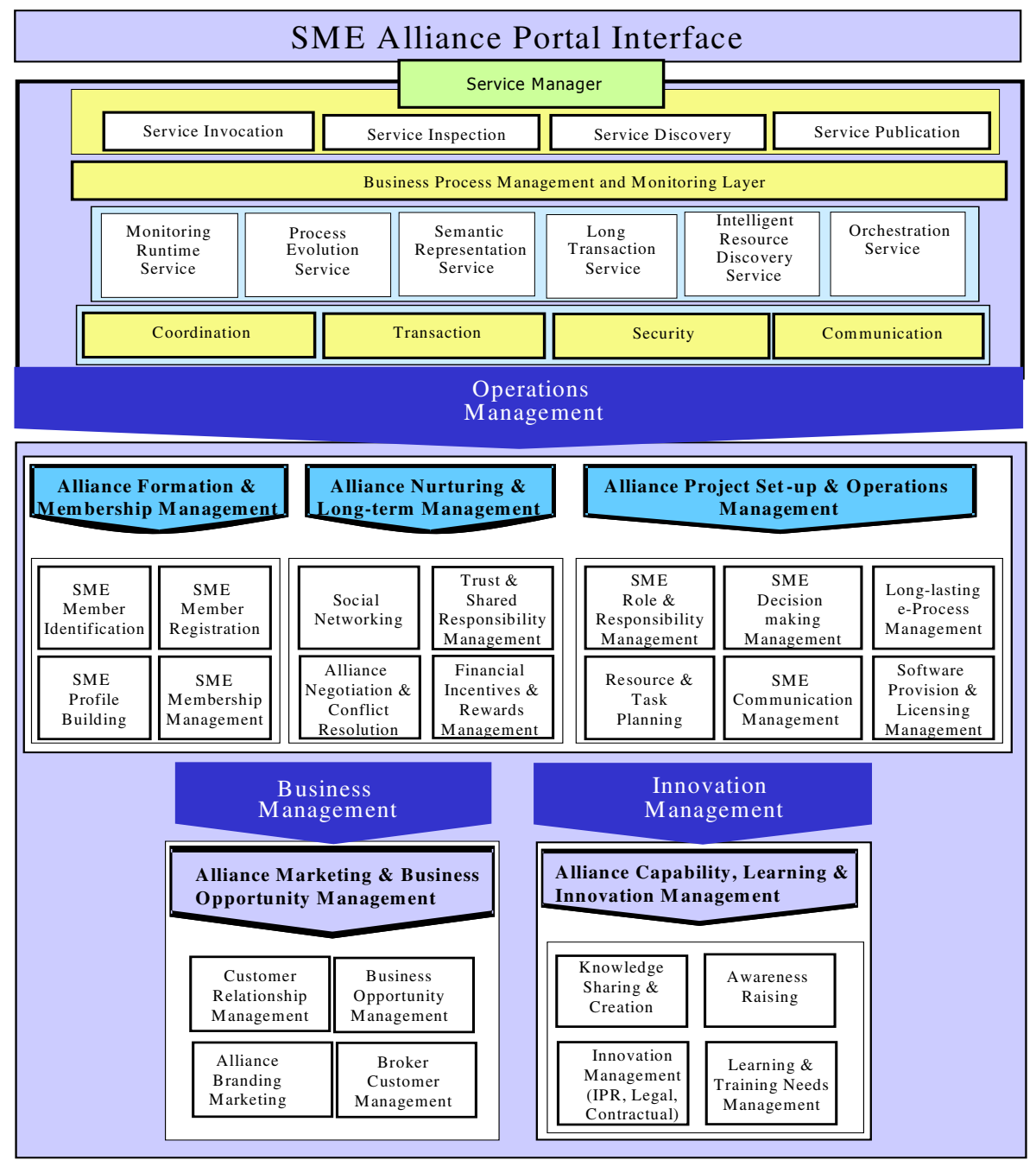

Fig. 1. The SME Alliance Portal Architecture

The proposed functionality is delivered using a service-oriented architecture. This is based on a set of loosely coupled components available as services, delivered using an Application Service Provider (ASP) like model [15]. Two main roles emerge in this model:

Alliance ePlatform Infrastructure Provider and Manager (Alliance ASP): This has the responsibility of managing the service infrastructure (e.g. servers, computer resources, etc.) and allocating shared workspace environments to potential Alliance clients to host their projects. This involves hosting the core infrastructure through the provision of and access to both core services 
and third party services (TPS). Core services refer to services necessary for the basic operation and management of services, including TPS that are provided by third party service providers. The Alliance ASP, through the core services, has the capability to host multiple alliance projects and to make available different services (both core and TPS) to various alliance projects. They can also play the role of application integrators, providing help and assistance to migrate legacy applications and legacy systems to web services.

$>$ Third party service providers (TPSP): These represent various companies, including software houses, interested in making their software application(s) accessible through a service-based middleware solution hosted and managed by the Alliance ASP. These companies have their services published within the ASP UDDI (Universal Description, Discovery, and Integration) registry. Typically, these services would fulfill a particular purpose for an alliance project. Examples of services offered by TPSP include structural dimensioning services, HVAC simulation service, procurement service, and facility management (FM) service.

However, a number of key issues emerge from the literature as essential to support effectively web services, including: Coordination (to manage interaction between services and coordination of sequences of operations, to ensure correctness and consistency); Transaction (to manage short-duration / atomic and long running business activities); Context (to adjust execution and output to provide the client with a customized and personalized behavior: may contain information such as a consumer's name, address, and current location, the type of client device, including hard- and software that the consumer is using, or preferences regarding the communication); Conversation modeling (to facilitate service discovery and dynamic binding, service composition model validation, service composition skeleton generation, analysis of compositions and conversations and conversation model generation); Execution monitoring (involves either centralized or distributed execution of composite web services). These limitations are discussed in the following section.

\section{Key Limitations and Proposed Research Directions}

Existing web service technology is ill suited to support the dynamic and changing nature of service environments. Key limitations emerge and have been reported, which hinder full exploitation of web services as a promising middleware technology, including:

- $\quad$ Existing service description and Web Service flow languages do not support effectively the dynamics and non-functional characteristics of distributed business processes. The current Business Process Execution Language (BPEL) version as described in [16] does not support run-time alterations to address unforeseen problems, such as the replacement or addition of a Web Service. In order to manage this uncertainty, BPEL processes need to have the ability to be extended to meet unforeseen post-deployment requirements and user needs. 
- Web service flow engines, such as the ones implemented to support BPEL, lack execution monitoring functionality to manage the running process. These can help debug processes during development stage, with monitoring, and may even be driven by agents at production stage. It is possible, for example, to embed, without modifying the engine implementation, a planner on the top of the latter. From events triggered by a monitor, this planner can take actions to avoid any disruption and to adjust the process. Such a tool can be useful particularly for long running processes.

- Web service composition methodologies have a focus on syntactic integration and therefore do not support automatic composition of web services. Semantic integration is crucial for web services as it allows them to (a) represent and reason about the task that a web service performs, (b) explicitly express and reason about business relations and rules, (c) understand the meaning of exchanged messages, (d) represent and reason about preconditions that are required to use the service and the effects of having invoked the service, and (e) allow intelligent composition of web services to achieve a more complex service.

The following sub-sections discuss potential research directions, with a focus on (a) business process description and management, (b) service discovery, (c) service composition, (d) service semantic compatibility management.

\subsection{Business Process Description and Management}

From a practice (industry) perspective, business processes are modeled as one-off exercise and assume that processes are static. If the requirements of the process change, then the entire specification is updated. The available business process description languages, such as BPEL, allow programmers to describe and implement complex web services as distributed processes and to compose them in a general way. However, the definition of new processes that interact with existing ones must be done manually, and this can be a technically demanding, time consuming, and error prone task. Additionally, in the current BPEL environment it is not possible at runtime, to add an unforeseen web service into the process, to replace Web services, or to hot-fix processes. The process needs to be stopped in order that it can be updated / extended.

\subsection{Service Discovery}

Mechanisms to easily locate services which meet the process requirements are necessary for both the service provider and the service requester. On the one hand, from the service provider standpoint, improved service reachability implies higher service use potential. On the other hand, the service requester needs to be able to find the most suitable services with respect to existing requirements. Publication, discovery and selection are based on a Service Directory, such as Universal Discovery Description and Integration [17]. The role of such a tool is to provide methods, which (a) can be used by the service provider to publish the provided services and (b) can be used by the service requester to look for the services, which satisfy his requirements. 
However, even if UDDI allows browsing of the service registry through different indexes, it does not provide:

$>\quad$ an effective content-based service discovery

$>$ a match with respect to the context in which the service operates

$>$ a quality evaluation of the retrieved services

\subsection{Service Composition}

There are theoretically two main paradigms which can be used to model business processes or build workflows by means of web services. These are either manual or automatic composition. In the manual approach, human users who know the domain well (e.g., domain ontology) select suitable web services and assemble them into a cohesive workflow. Although users may use graphical user interface based software to facilitate the composition, in essence, it is a labor-intensive and error-prone task and thus is not seen as appropriate or efficient for large-scale composition problems.

However, in the automatic composition approach, it is assumed that software programs know if two web services can be connected or not (i.e., via syntactic matching of web services parameters or possibly via semantic matching). Major Web and Semantic Web services composition languages include: BPEL4WS, BPML, WSCI, WSCDL and DAML-S. These standards all support the imperative elements of service composition, e.g. exception handling and compensation and all possess the capability to compose more complex structures and activities.

Business collaborations require the enforcement of business agreement on quality of service (QoS). However these languages do not all address this. BPEL has gained the widest support from industry and most major software vendors have pledged BPEL support in their products. Current web service composition frameworks include eFlow [18], MAIS, MOEM, SELF-SERV [19], OntoMat-Service [20], SHOP2 [21], WebTransact [22] and StarWSCoP [23].

The current state of the development effort is generally centered on attempts to create service composition platforms, since the current state of research lacks additional specifications concerning quality of service extensions in Web Services Description Language (WSDL), to name a simple but crucial example, and some platforms have developed their own proprietary data structures for extending WSDL.

\subsection{Service Semantic Compatibility Management}

Since individual web services are created in isolation, a key disadvantage is that there are often many issues related to the use of abbreviations, different formats, or typographical errors embedded within their vocabularies. For example, two terms with different spellings or abbreviations may have the same semantic meaning and are thus inter-changeable. Diverse matching schemes have been developed to address these semantic resolution problems, including through the use of ontologies [24]. In general, matching approaches generally fall into three categories:

$>$ Exact matching using syntactic equivalence.

$>$ Approximate match using distance functions (e.g. TF-IDF, Jaccard, SoftTFIDF, Jaro, or Levenstein distance).

$>$ Semantic matching using ontologies [25]. 
Research within the Semantic Web Community proposes a top-down, unambiguous description of service capability, e.g. in DAML-S, which allows for the automation of web services tasks such as discovery and composition through reasoning.

\section{Conclusion}

The paper has identified a set of functionality as well as key limitations in the area of dynamic web service based process modeling and execution to support alliance modes of operation of SMEs in the Construction sector. A call is made to further research in this area, including, on the one hand, developing solutions for transient SME alliances, and on the other, developing adapted solutions that support run-time adaptable and extensible service-based e-processes.

As argued above, the use of web service technologies is hampered by the static nature of the languages used to model business processes, which cannot easily adapt and allow for changes in processes and service components.

Therefore, some essential support features for designing and developing composite services are required. These include execution-monitoring capabilities to manage and trace service execution, dynamic service selection, quality of service (QoS) modelling and evaluation, dynamic transaction and coordination support, and the ability to dynamically model service flows.

The paper therefore argues that there would be great value in investigating the extension of BPEL environments towards more flexibility, agility and self-adaptability not only regarding the discovery and activation of services, but mainly in the context of long-lasting business processes, whose life span is much longer than the typical life span of a service provider or business partner. The networked enterprise software must be adapted to accommodate new features in the context of changing requirements. As BPEL is an extensible language (new instructions can be added to take account of user's evolving and changing needs), its engine should be extensible to integrate new behaviors for user-specific instructions (including instructions to replace an existing service in accordance with run-time criteria and context).

Future research should strive to illustrate how these applications with aspect weaving capabilities could be dynamically adapted to allow for unforeseen features. Aspects should be considered and employed at three levels:

$>$ In the context of semantic analyzers.

$>$ Within a BPEL engine that orchestrates Web Services.

$>$ Within BPEL processes themselves.

Each level should use its own tailored Domain-Specific Aspect Language (DSAL) that should be easier to manipulate than a general purpose one (close to the programming language) with the pointcuts being independent from the implementation.

Research should create advances by addressing new requirements to the classical service-oriented approach, in particular:

$>$ The ability to both abstract service characteristics from their operating environment and in some cases select services on the basis of their contextual features. 
The ability to select services both through interactive interfaces and through sophisticated matching algorithms.

Further research should strive to make advances by improving service semantic compatibility resolution and matching using ontologies (e.g., RDF and OWL). The latter should take a pivotal role in the process by checking semantic relatedness between data structures and / or concepts used by each partner service. It is essential in this context to handle uncertainty in the underlying reasoning by drawing on fuzzy concept research. Fuzzy concept techniques should offer the means to address these uncertainty aspects and should constitute real advances in the field of semantic service composition.

It is hoped that the paper has provided useful insights into the area of service-based business process execution research, and that this will trigger further research with promising potential.

\section{References}

[1] Dick, J., Payne, D.: Regional Sectoral Support: A review of the construction industry, SMEs and regional innovation strategies across Europe. International Journal of Strategic Property Management 9, 55-63 (2005)

[2] Abulma'thi, A., Rezgui, Y.: Virtual Organizations in Practice: A European Perspective. In: 14th Americas Conference on Information Systems (AMCIS 2008), Toronto, Ontario, August 14-17 (2008)

[3] Rezgui, Y., Zarli, A.: Paving the way to digital construction: a strategic roadmap. Journal of Construction Engineering and Management (Journal of the American Society of Civil Engineers) 132(12), 767-776 (2006)

[4] Powell, A., Piccoli, G., Ives, B.: Virtual teams: a review of current literature and directions for future research. The Database for Advances in Information Systems 35(1), 6-36 (2004)

[5] Jarvenpaa, S., Leidner, D.: Communication and trust in global virtual teams. Organization Science 10(6), 791-815 (1999)

[6] Lipnack, J., Stamps, J.: Virtual Teams: People Working Across Boundaries with Technology, 2nd edn. Wiley, New York (2000)

[7] Camarninha-Matos, L., Afsarmanesh, H., Ollus, M. (eds.): Virtual organizations: systems and practice. Springer Science, New York (2005)

[8] Goranson, H.T.: The Agile Virtual Enterprise: Cases, Metrics, Tools, Quorum Books, Westport, CT (1999)

[9] Rezgui, Y., Miles, J.C.: Transforming SME strategies via innovative transient knowledge-based alliances in the Construction Sector. In: INDIN 2009, 7th IEEE Conference on Industrial Informatics, Cardiff, June 24-26 (2009)

[10] Stal, M.: Web services: beyond component-based computing. Commun. ACM 45(10), 71-76 (2002)

[11] Zeng, L., Benatallah, B., Ngu, A.H.H., Dumas, M., Kalagnanam, J., Chang, H.: QoSAware Middleware for Web Services Composition. IEEE Transactions on Software Engineering 30(5), 311-327 (2004)

[12] Rezgui, Y.: Role-Based Service-Oriented Implementation of a Virtual Enterprise: A Case Study in the Construction Sector. Computers in Industry 58(1), 74-86 (2007) 
[13] Rezgui, Y., Medjdoub, B.: A Service Infrastructure To Support Ubiquitous Engineering Practices. In: Establishing the foundation of collaborative networks, pp. 627-636. Springer, Heidelberg (2007)

[14] McIlraith, S.A., Son, T.C., Zeng, H.: Semantic Web Services. IEEE Intelligent Systems 16(2), 46-53 (2001)

[15] Papazoglou, M.P.: Service-oriented computing: concepts, characteristics and directions. In: WISE 2003. Proceedings of the Fourth International Conference on Web Information Systems Engineering, pp. 3-12 (2003)

[16] Leymann, F., Roller, D., Schmidt, M.-T.: Web services and business process management. IBM Systems Journal 41(2), 198-211 (2002)

[17] Menascé, D.A.: QoS Issues in Web Services. IEEE Internet Computing 6(6), 72-75 (2002)

[18] Rezgui, Y.: Exploring Virtual Team-Working Effectiveness in the Construction Sector. Interacting with Computers 19(1), 96-112 (2007)

[19] OASIS, BPEL specification, http: / /www.oasis-open.org/committees / tc_home.php?wg_abbrev=wsbpel (last accessed 05/04/09)

[20] OASIS, UDDI specification, http: / / www. oasis-open.org/committees / tc_home.php?wg_abbrev=uddi-spec (last accessed 05/04/09)

[21] Casati, F., Ilnicki, S., Jin, L., Vasudev Krishnamoorthy, V., Shan, M.-C.: Adaptive and Dynamic Service Composition in eFlow, HP (2000), http: / /www.hpl.hp.com/techreports / 2000/HPL-2000-39.pdf (last accessed 04/04/09)

[22] Benatallah, B., Sheng, Q.Z., Dumas, M.: The Self-Serv environment for Web services composition. IEEE Internet Computing 7(1), 40-48 (2003)

[23] Onto-Mat website, http: //www.aifb.uni-karlsruhe.de/mailman/listinfo/ontomat (last accessed 04/04/09)

[24] Nau, D., Au, T.-C., Ilghami, O., Kuter, U., Murdock, J.W., Wu, D., Yaman, F.: SHOP2: An HTN Planning System. Journal of Artificial Intelligence Research 20, 379-404 (2003)

[25] Pires, P.F., Benevides, R.F.M., Mattoso, M.: WebTransact: A Framework for Specifying and Coordinating Reliable Web Service Compositions. Technical Report ES-578/02 PESC/Coppe Federal University of Rio de Janeiro (2002),

http: / / www.cos.ufrj.br/ pires/webTransact.html (last accessed 04/04/09)

[26] Sun, H., Wang, X., Zhou, B., Zou, P.: Research and Implementation of Dynamic Web Services Composition. In: Zhou, X., Xu, M., Jähnichen, S., Cao, J. (eds.) APPT 2003. LNCS, vol. 2834, pp. 457-466. Springer, Heidelberg (2003)

[27] Barresi, S., Rezgui, Y., Celson, L., Meziane, F.: Architecture to Support Semantic Resources Interoperability. In: Proceedings of the ACM workshop on Interoperability of Heterogeneous Information Systems (IHIS 2005), Germany. ACM Press, New York (2005)

[28] Rezgui, Y., Nefti-Meziani, S.: Ontology-Based Dynamic Composition of Services Using Semantic Relatedness and Categorisation Techniques. In: Proceedings of the 9th International Conference on Enterprise Information Systems (ICEIS), Funchal, Madeira - Portugal, June 12-16 (2007) 